\title{
Detection of upper gastrointestinal cancer in patients taking antisecretory therapy prior to gastroscopy
}

\author{
M G Bramble, Z Suvakovic, A P S Hungin
}

\begin{abstract}
Background-The incidence of early gastric cancer has not increased despite better access to endoscopic facilities for general practitioners. Many patients receive a course of symptomatic treatment while waiting for gastroscopy.

Aims-To ascertain the effect of antisecretory therapy on the diagnostic process and findings for patients with upper gastrointestinal cancer.

Methods-A consecutive case study survey of the primary care records of 133 patients who had died of upper gastrointestinal cancer during 1995-97 in the South Tees health district in the northeast of England (population 300 000).

Results-From the 133 patients identified, $\mathbf{1 1 6}$ had died from adenocarcinoma of the oesophagus (31) or stomach (85). Failure to reach the diagnosis of cancer at the index gastroscopy was associated with prior acid suppression therapy. Only one of 54 patients on no treatment or antacids alone was erroneously diagnosed as suffering from benign disease, whereas 22 of 62 patients treated with acid suppression were diagnosed as suffering from benign disease but at varying times later turned out to have adenocarcinoma. Twenty of 45 patients taking a proton pump inhibitor had a delayed diagnosis compared with two of 17 taking an $\mathrm{H}_{2}$ receptor antagonist. The commonest lesion seen at index gastroscopy in those in whom the diagnosis was initially missed was gastric ulcer. Healing occurred in six patients taking a proton pump inhibitor, despite their later diagnosis of malignancy.

Conclusions-The treatment of dyspeptic symptoms with acid suppression prior to gastroscopy masks and delays the detection of gastric and oesophageal adenocarcinoma on endoscopy in one third of patients.

(Gut 2000;46:464-467)
\end{abstract}

Keywords: diagnosis; upper gastrointestinal cancer; gastric adenocarcinoma; oesophageal adenocarcinoma; gastroscopy; antisecretory therapy

Centre for Health Studies, University of Durham, UK

A P S Hungin

Correspondence to: Dr M G Bramble

Accepted for publication 22 October 1999

The prognosis of patients with oesophagogastric cancer is heavily dependent on disease stage at the time of diagnosis. ${ }^{1}$ In the United Kingdom good results from surgery are possible $^{2}$ although the overall benefits from surgery have not been realised because the majority of patients in this country are diagnosed with advanced stage disease (greater than stage I). Less than a third of patients survive beyond three years, even when the disease is potentially curable by radical surgery. ${ }^{3}$ Open access gastroscopy has the potential for improving the detection rate of earlier stage disease but the majority of patients with gastric cancer are not referred through this channel and the overall incidence of early stage disease remains depressingly low. ${ }^{4}$ A possible contributing factor is the use of acid suppression therapy prior to diagnostic gastroscopy. General practitioners requesting gastroscopy, through open access or by consultant referral, may feel pressurised to provide effective symptom control with acid suppression therapy if waiting times to investigation are long, even in patients over 45 years of age. ${ }^{5}$

The aim of this study was to quantify the risk of such a strategy in one typical health district.

\section{Patients and methods}

The study was set in the South Tees health district of Teesside, a mixed industrial and rural area with a catchment population of 300000 . Owing to the centralisation of gastrointestinal services it is estimated that over $95 \%$ of all patients referred with gastrointestinal problems are seen at one hospital. This enabled centrally available access to gastroscopy and pathology, including histology reports.

For this study the general practice case records of all patients who had died from oesophagogastric cancer over the three year period from 1995 to 1997 were studied. These patients were initially identified from hospital pathology results and their records were accessed on their death. The records were used to ascertain factors leading to the initial hospital referral for investigation by gastroscopy, including the time elapsed to investigation, any history of prior acid suppression therapy prescribing and any subsequent association between the use of acid suppression therapy and the diagnostic process. For analysis of results patients were categorised into two groups: those who had been prescribed acid suppression therapy prior to gastroscopy and those who had not. Acid suppression therapy was defined as the use of any $\mathrm{H}_{2}$ receptor antagonist $\left(\mathrm{H}_{2} \mathrm{RA}\right)$ or proton pump inhibitor
(PPI) during the six months prior to the initial (index) gastroscopy.

Abbreviations used in this paper: PPI, proton pump inhibitor; $\mathrm{H}_{2} \mathrm{RA}, \mathrm{H}_{2}$ receptor antagonist. 
Table 1 Patients with adenocarcinoma of the oesophagus not detected at the index gastroscopy

\begin{tabular}{llllll}
\hline Endoscopy result & Age & Sex & Treatment & Duration & Delay \\
\hline Gastric ulcer & 81 & $\mathrm{~F}$ & PPI & 24 weeks & 1 year \\
Duodenal ulcer & 68 & $\mathrm{M}$ & $\mathrm{H}_{2} \mathrm{RA}$ & 2 years & 6 months \\
Barrett's oesophagus & 85 & $\mathrm{M}$ & $\mathrm{PPI}$ & 5 years & 2 months \\
Normal & 56 & $\mathrm{M}$ & PPI & 8 weeks & 3 years \\
Oesophagitis & 52 & $\mathrm{M}$ & Antacids & & 3 months \\
Oesophagitis & 87 & $\mathrm{~F}$ & PPI & 5 years & 3 months \\
\hline
\end{tabular}

Mean age of patients 69.8 years (range $52-87$ ).

PPI, proton pump inhibitor; $\mathrm{H}_{2} \mathrm{RA}, \mathrm{H}_{2}$ receptor antagonist.

Table 2 Patients with adenocarcinoma of the stomach not detected at the index gastroscopy

\begin{tabular}{llllll}
\hline Endoscopy result & Age & Sex & Treatment & $\begin{array}{l}\text { Duration of } \\
\text { treatment }\end{array}$ & Delay to diagnosis \\
\hline Gastric erosion & 58 & $\mathrm{M}$ & PPI & $4-8$ weeks & 2 months \\
Oesophagitis & 85 & $\mathrm{~F}$ & PPI & $12-24$ weeks & 2 months \\
Duodenal ulcer & 81 & $\mathrm{~F}$ & PPI & $12-24$ weeks & 3 months \\
Duodenal ulcer & 62 & $\mathrm{M}$ & PPI & $24-52$ weeks & 24 months \\
Gastric ulcer & 92 & $\mathrm{~F}$ & PPI & $24-52$ weeks & 5 months \\
Gastric ulcer & 97 & $\mathrm{M}$ & PPI & $4-8$ weeks & 4 months \\
Gastric ulcer & 63 & $\mathrm{~F}$ & PPI & $4-8$ weeks & 6 months \\
Gastric ulcer & 43 & $\mathrm{M}$ & PPI & $4-8$ weeks & 3 months \\
Gastric ulcer & 86 & $\mathrm{~F}$ & PPI & 3 years & 2 years \\
Gastric ulcer & 38 & $\mathrm{~F}$ & PPI & 2 years & 4 years \\
Gastric ulcer & 68 & $\mathrm{~F}$ & H RA & $4-8$ weeks & 2 years \\
Gastric ulcer & 81 & $\mathrm{M}$ & PPI & $12-24$ weeks & 5 months \\
Gastric ulcer & 71 & $\mathrm{M}$ & PPI & 5 years & 3 years \\
Gastric ulcer & 75 & $\mathrm{M}$ & PPI & $24-52$ weeks & 4 years \\
Hiatus hernia & 74 & $\mathrm{~F}$ & PPI & 5 years & 6 months \\
Hiatus hernia & 74 & $\mathrm{~F}$ & PPI & 3 years & 2 years \\
Normal & 79 & $\mathrm{M}$ & PPI & 24-52 weeks & 4 years \\
\hline
\end{tabular}

Mean age of patients 72.1 years (range 38-97). M:F $=8: 9$.

Suspected malignant ulcers but with "normal" biopsies are not included in the table. PPI, proton pump inhibitor; $\mathrm{H}_{2} \mathrm{RA}, \mathrm{H}_{2}$ receptor antagonist.

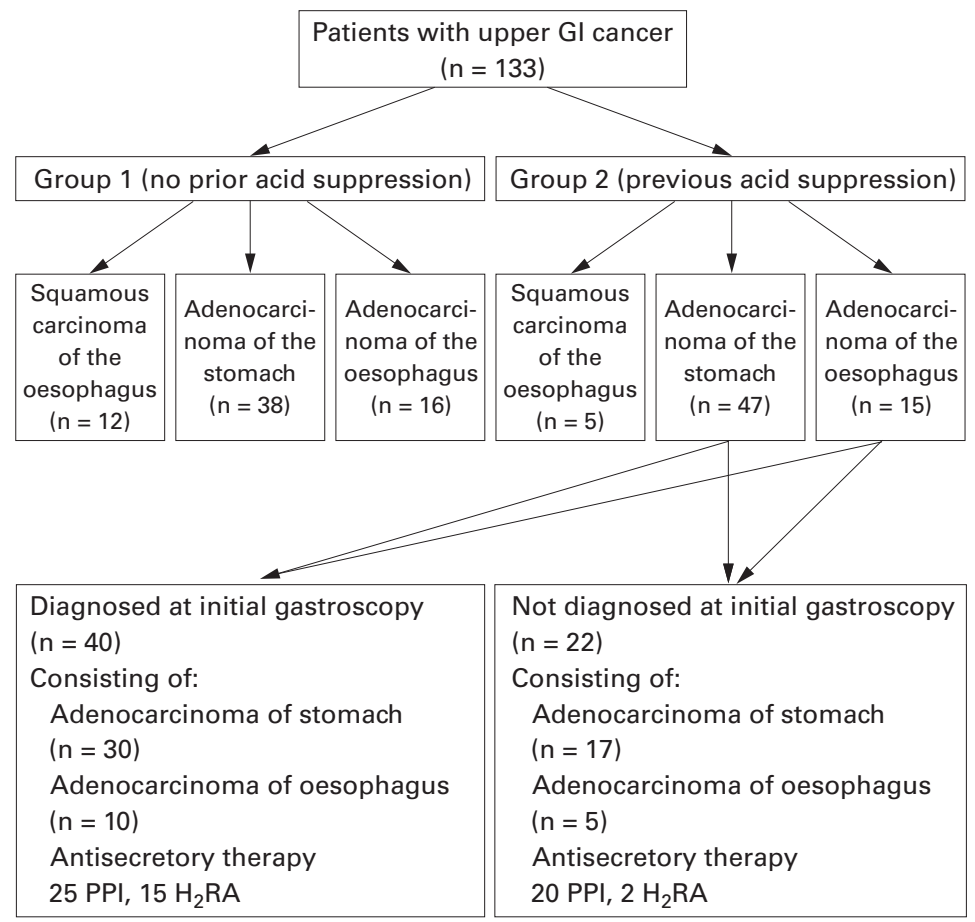

Figure 1 Diagnosis and investigations in 133 patients with upper gastrointestinal cancer.

The gastroscopy reports were available from a computerised database and the original paper records. The general practice records were retrieved by a researcher (ZS) from the practices or from the central registry of the Health Authority if the records had been recalled following the patients' deaths. Data were maintained on a PC based spreadsheet and results compared, where applicable, using the $\chi^{2}$ test with $\mathrm{p}$ values ( $5 \%$ significance, $95 \%$ confidence limits, one degree of freedom).

\section{Results}

A total of 133 patients were discovered to have died from cancer of the stomach or the oesophagus (fig 1). Of these, 85 (64\%) had gastric adenocarcinoma, 31 (23\%) oesophageal adenocarcinoma, and 17 (13\%) squamous cell oesophageal carcinoma (tables 1 and 2). Seventy patients were male and the mean age of the whole group was 69 years (range 38-97). Sixty seven (50\%) had been prescribed acid suppression therapy prior to the initial (index) gastroscopy.

\section{SQUAMOUS CELL CARCINOMA OF THE} OESOPHAGUS

Seventeen patients were diagnosed as suffering from a squamous cell carcinoma of the oesophagus. Five of these had been prescribed acid suppression therapy (four PPI, one $\mathrm{H}_{2} \mathrm{RA}$ ) prior to investigation. In one patient the diagnosis was not made at the index gastroscopy.

ADENOCARCINOMA OF THE OESOPHAGUS

Of 31 patients with this eventual diagnosis 15 (48\%) had received acid suppression therapy prior to gastroscopy (five $\mathrm{H}_{2} \mathrm{RA}, 10 \mathrm{PPI}$ ). In five patients the lesion was not detected at index gastroscopy and all were found to have been on acid suppression therapy (one $\mathrm{H}_{2} \mathrm{RA}$, four PPI). In contrast, of 16 patients not on any prior acid suppression therapy, all but one was diagnosed at the index gastroscopy.

\section{ADENOCARCINOMA OF THE STOMACH}

Eighty five patients were eventually diagnosed as having gastric adenocarcinoma. Of these, 47 $(55 \%)$ were prescribed acid suppression therapy prior to the index gastroscopy. The diagnosis was made at index gastroscopy in all 38 patients not taking prior acid suppression therapy compared with 30 of the $47(63 \%)$ on prior acid suppression therapy $\left(\chi^{2}=14.99\right.$, $\mathrm{p}<0.0002)$. In the 17 patients not diagnosed at the index gastroscopy the mean delay to diagnosis was 13 months (range 2-36). As a high proportion of patients had started acid suppression therapy several months prior to the index gastroscopy the mean delay from inception of therapy to cancer diagnosis was 26 months (range 4-60), excluding two patients who been taking acid suppression therapy for more than three years.

The most frequently occurring "erroneous" diagnosis was gastric ulcer (10 patients). In six patients, complete or near complete healing of their gastric ulcers, associated with negative biopsies at the time of repeat gastroscopy, was noted.

Of the 68 patients classified as having cancer from the index gastroscopy, $23(32 \%)$ had, in fact, undergone investigation before, although in all cases, more than five years previously. Fourteen of these had been prescribed acid suppression therapy prior to the index gastroscopy (seven $\mathrm{H}_{2} \mathrm{RA}$, seven PPI) on a regular or intermittent basis over many years. Sixteen of 
the remaining 45 patients who had not undergone any investigation prior to the index gastroscopy were also prescribed acid suppression therapy, with 12 taking PPIs.

ALL UPPER GASTOINTESTINAL ADENOCARCINOMAS Overall, a total of 116 patients had upper gastrointestinal adenocarcinoma. One of 54 patients $(2 \%)$ not on acid suppression therapy prior to gastroscopy was erroneously diagnosed as suffering from benign disease compared with 22 of 62 patients $(35 \%)$ on acid suppression therapy $\left(\chi^{2}=18.48, \mathrm{p}<0.00002\right)$.

Of the 62 patients with upper gastrointestinal adenocarcinoma who were on acid suppression therapy prior to the index endoscopy, $45(73 \%)$ were prescribed a PPI and 17 $(27 \%)$ an $\mathrm{H}_{2}$ RA. No data were available regarding the use of $\mathrm{H}_{2} \mathrm{RAs}$ bought over the counter. Overall, 67 patients (including 62 with adenocarcinoma) from the total of 133 had been prescribed acid suppression therapy and in 22 patients $(33 \%)$ the adenocarcinoma was not diagnosed at the index gastroscopy. The risk of not detecting the true nature of endoscopically observed lesions or of not seeing any pathology at all was greater in patients prescribed PPIs (20/45, 44\%) compared with $\mathrm{H}_{2} \mathrm{RAs}\left(2 / 17,12 \% ; \chi^{2}=4.42\right.$, $\mathrm{p}<0.05)$.

\section{Discussion}

This was a primary care based survey of patients referred to hospital through a variety of channels, for further investigation and management of dyspeptic symptoms, of whom $50 \%$ had already been prescribed acid suppression therapy, presumably for symptom relief. We do not know which factors led the general practitioner to treat some patients and not others. A potential flaw in the study is that some selection bias, such as the general practitioner's diagnosis, may have been affecting the decision to prescribe acid suppression therapy prior to gastroscopy. For example, patients with simple dyspepsia and no "alarm" symptoms might have been prescribed acid suppression therapy while waiting for gastroscopy to exclude the possibility of malignancy, while patients with suspected cancer were "fast tracked" for urgent investigation and not given acid suppression therapy. Unfortunately it is the group without alarm symptoms who have the most to gain by early diagnosis. This means that general practitioners (GPs) are treating precisely the group who will benefit most by not being prescribed acid suppression therapy and undergoing early investigation.

The waiting time for open access gastroscopy during the study period was four to 10 weeks, similar to the wait for an outpatient appointment. Most GPs are likely to consider 10 weeks too long a period to deprive a patient of effective symptom relief and the use of acid suppression therapy is understandable. However, the use of acid suppression therapy prior to gastroscopy uncovered two difficulties. Firstly, the diagnosis may have been masked in some patients whose lesions appeared benign (particularly gastric ulcers, with confirmatory biopsy results), and who continued on acid suppression therapy after the endoscopy, until the malignancy became manifest. Secondly, some patients were discovered at the index gastroscopy to have only minor abnormalities or normal results, but were later found to have late stage malignancies when re-examined some months or years later. These problems are unlikely to have been owing to differences in detection rates or observer errors as no patients with gastric cancer were "missed" in that group of patients not prescribed acid suppression therapy. Furthermore, the effect was more notable with PPIs which are known to produce more profound acid suppression than $\mathrm{H}_{2}$ RAs. Even if the alternative explanation that "ulcer-cancer" patients are more likely to receive acid suppression therapy is entertained, this still raises serious questions as to the appropriateness of acid suppression therapy as symptomatic treatment in this age group.

Previous reports about $\mathrm{H}_{2}$ RAs "masking" gastric cancer ${ }^{6}$ have largely been ignored in clinical practice. In line with findings from other centres, ${ }^{7}$ in our locality the diagnosis of gastric cancer has been reported as being delayed in up to $16 \%$ of patients. ${ }^{4}$ The majority of these patients $(88 \%)$ were on prior $\mathrm{H}_{2}$ RAs. This study shows that the corresponding figure for delayed diagnosis in patients on prior PPIs is in the region of $44 \%$. It is likely that the increasing use of PPIs in primary care ${ }^{8}$ has now made the problem more apparent. It is questionable whether all these patients had early gastric cancer at the time of the index gastroscopy. Although their biopsies showed no evidence of malignancy, the course of subsequent events was in keeping with the history of patients with developing gastric cancer. ${ }^{9}$ However, many patients returned to clinical care within a relatively short space of time, essentially a few months, suggesting that a significant number were harbouring cancer for a considerable time, even when their biopsies had been negative. The likeliest explanation is that acid suppression therapy promoted significant ulcer healing and mimicked a benign visual appearance. Biopsies of the healing edges of such ulcers do not necessarily contain malignant tissue because acid suppression therapy can promote overgrowth of normal mucosa in sites of malignant tissue. ${ }^{10}$ Ideally, six to eight biopsy specimens should be taken from any "suspicious" looking ulcer, ${ }^{11}$ but in practice the endoscopist may be content with fewer specimens if the ulcer looks benign. There were no differences in the biopsy policies in the patient groups in this study and those with gastric ulcers underwent further endoscopies and biopsies. In six patients the gastric ulcer had either healed completely or was significantly smaller after six to eight weeks of treatment (usually the continuation of a PPI). This is in accordance with recent endoscopic evidence that early malignancy within the gastric mucosa may heal with acid suppression therapy, particularly PPIs. ${ }^{10}$ This mechanism is likely to have been responsible for the ultimately erroneous endoscopic and histological appearance of the gastric ulcers in this study. The 
results do not throw any light on the question as to whether acid suppression therapy might cause upper gastrointestinal cancer. However, it is now accepted that one of the main indications for this type of treatment, namely gastrooesophageal reflux disease, is in itself a risk factor for oesophageal and gastric cardia adenocarcinoma. ${ }^{12}$

This study shows that prior acid suppression therapy makes it more difficult for the endoscopist to reach an early and correct diagnosis even when macroscopic pathology, such as gastric ulcer, is noted. We propose that clinical practice guidelines should incorporate advice on avoiding or ceasing acid suppression therapy for a significant period prior to gastroscopy. Although the consequences of not visualising a benign peptic ulcer or mucosal lesion may not be dire, the consequences of a delay in the diagnosis of cancer, particularly early gastric cancer, are more significant. It is not clear in the light of present knowledge how long patients should cease acid suppression therapy to enhance the detection of cancer early and accurately, but this is likely to be considerably longer than the two to four weeks proposed by most gastroenterologists aiming to increase their yield of peptic ulcers.

While such advice needs to be heeded by both GPs and hospital clinicians, this research reiterates the need for short waiting times to gastroscopy for patients considered to be at increased risk of gastric cancer and places an onus on hospital services to improve gastroscopy waiting times. The responsibility for managing such patients is joint between primary and secondary care and exhortations to avoid acid suppression therapy prescribing in say, older dyspeptic patients, is of limited value if early investigation is not possible. If one of the goals of providing gastroscopy services is to improve the detection rate of early gastric cancer it is probably counter productive to allow a situation where patients are deprived of effective symptom relief, usually with antisecretory drugs, for more than four weeks. In the ideal setting patients considered at increased risk of cancer, for example, by nature of age or symptoms should have no prior acid suppression therapy and should proceed to an early examination characterised by multiple biopsies.

\section{Addendum}

Since submitting this study for publication all the remaining biopsy material from the gastric ulcer patients (embedded in paraffin wax and available for further analysis) has been subject to further examination by two independent histopathologists. One ulcer was considered to contain malignant cells (carcinoma in situ) by both pathologists but the remaining biopsies from all the other ulcers showed no evidence of malignancy.

We would like to thank Professor N A Wright and Dr R A Jones for re-examining the biopsy material from all the gastric ulcer patients.

Conflict of Interest Statement: A P S Hungin has provided remunerated educational input for Wyethlederle, Astra and Glaxo.

1 Allum WH, Powell DJ, McConkey CC, et al. Gastric cancer. A 25 year review. Br F Surg 1989;76:535-40.

2 Sue-Ling HM, Johnson D, Martin IG, et al. Gastric cancer: a curable disease in Britain. BMF 1993;307:591-6.

3 Cuscheri A, Fayers P, Fielding J, et al. Postoperative morbidity and mortality after D1 and D2 resections for gastric cancer: preliminary results or the MRC randomised controlled surgical trial. Lancet 1996;347:995-9.

4 Suvakovic Z, Bramble MG, Jones R, et al. Improving the detection rate of early gastric cancer requires more than detection rate of early gastric cancer requires more than
open access gastroscopy. A five year study. Gut 1997;41: open access

5 Axon ATR, Bell GD, Jones RH, et al. Guidelines on appropriate indications for upper gastrointestinal endoscopy. BMF 1995;310:853-6.

6 Taylor RH, Lovell D, Menzies-Gow N, et al. Misleading response of malignant ulcers to cimetidine. Lancet 1978;i: 686-8.

7 Martin IG, Young S, Sue-Ling HM, et al. Delays in the diagnosis of oesophagogastric cancer: a consecutive case series. BMF 1997;314:467-71.

8 Martin RM, Lim AG, Kerry SM, et al. Trends in prescribing H2-receptor antagonists and proton pump inhibitors in primary care. Aliment Pharmacol Ther 1998;12:797-805.

9 Tsukuma H, Mashima T, Oshima A. Prospective study of early gastric cancer. Int $\mathcal{F}$ Cancer 1983;31:421-6.

10 Wayman J, Hayes N, Biggin CS, et al. Response of early gasWayman J, Hayes N, Biggin CS, et al. Response of early gas-
tric cancer to proton pump inhibitors. N Engl f Med 1998; tric cancer to

11 Cotton PB, Williams CB. Diagnostic upper endoscopy. In: Practical gastrointestinal endoscopy. Oxford and London: Blackwell Scientific Publications, 1990:46-9.

12 Lagergren J, Bergstrom R, Lindgren A, et al. Symptomatic gastroesophageal reflux disease as a risk factor for esophageal carcinoma. $N$ Engl f Med 1999;340:825-31. 